\title{
Mixed Ligand, Palladium(II) and Platinum(II) Complexes of Tertiary DiphosphineswithS-1H Benzo[d] Imidazole-2-yl Benzothioate
}

\author{
KARWAN OMER ALI', HIKMAT ALI MOHAMMAD', \\ THOMAS GERBER ${ }^{3}$ and ERIC HOSTEN ${ }^{3}$
}

${ }^{1}$ College of Education and Human Sciences, university of Halabja, Halabja, Iraq.

${ }^{2}$ Department of Chemistry, college of Education, university of Salahaddin, Erbil.

${ }^{3}$ Department of Chemistry, Nelson Mandela Metropolitan University,

Port Elizabeth, 6031, South Africa.

*Corresponding author E-mail: Thomas.Gerber@nmmu.ac.az

HTTP://DX.DOI.ORG/10.13005/OJC/330205

(Received: October 24, 2016; Accepted: January 01, 2017)

\begin{abstract}
Palladium(II) and platinum(II) complexes containing the mixed ligands tertiary diphosphinesdppm. dppp and dppf with Thioester ligand S-1H benzo[d] imidazole-2-yl benzothioate (HSBIBT) have been prepared by the reaction of $\mathrm{PdCl}_{2}$ and $\mathrm{PtCl}_{2}$ with one equiv of tertiary diphosphines ligands to form $\left[\mathrm{Pd}\left(\mathrm{k}^{2}-\mathrm{dppf}\right) \mathrm{Cl}_{2}\right],\left[\mathrm{Pd}\left(\mathrm{k}^{2}-\mathrm{dppp}\right) \mathrm{Cl}_{2}\right]$ and $\left[\mathrm{Pt}\left(\mathrm{k}^{2}-\mathrm{dppmCl}\right) \mathrm{Cl}_{2}\right]$ complexes and then add the ligand HSBIBT to these complexes to form mixed ligand complexes. The prepared complexes have been characterized by single-crystal $X$-ray diffraction, elemental analysis, magnetic susceptibility, molar conductance, IR spectral data and UV-Visible. The results suggested that the ligand HSBIBT bonded to the metal through $\mathrm{N}$ atom and square planner geometries were assigned for the complexes.
\end{abstract}

Keywords: Palladium,Platinum,Tertiarydiphosphine,Thioester ligand complexes.

\section{INTRODUCTION}

Benzimidazole is the heterocyclic aromatic organic compound ${ }^{1}$. This bicyclic ring system consist of benzene ring joined with 4- and 5- situation of imidazole ring, imidazole ring having non adjacent two nitrogen atoms ${ }^{2}$. 2-Mercaptobenzimidazole derived from benzimidazole with thiol group in the 2-position. It holds extra chemical names such as, o-phenylenthiourea, benzimidazol-2-thion with formula of $\mathrm{C}_{7} \mathrm{H}_{6} \mathrm{~N}_{2} \mathrm{~S}^{3,4}$. In 2-mercaptobenzimidazole have two $\mathrm{N}$ atoms and one $\mathrm{S}$ atom and they can independently contribute to its binding to metal. 2-mercaptobenzimidazole derivatives, one of 
the most important derivatives of benzimidazole exhibited a wide range of importance biological activities such as antimicrobial ${ }^{6}$, antihistamine ${ }^{7}$, neutropic ${ }^{8}$ and analgesic ${ }^{9}$, antiprotozoal, antiviral, and anticancer activities ${ }^{10}$. Metal derivatives of heterocyclic-2-thiones have shown a variety of biochemical application: as antidandruff, antifungal, antibacterial, antibiotic and in shampoos, hair creams, plant diseases and hepatitis ${ }^{11}$.

Thioesters are important class of organosulfur compounds that play a major role in the construction of pharmaceutical, biological, industrial and natural products. In this respect, thioesters synthesis is one of the most important tasks in organosulfur chemistry. The esterification of thiols is the best and most common strategy for the synthesis of Thioesters ${ }^{12}$. Diphosphines are a class of chelating ligands that contain two phosphine groups connected by a bridge (also referred to as a backbone). The bridge, for instance, might consist of one or more methylene groups or multiple aromatic rings with heteroatoms attached ${ }^{13}$. The structure of the backbone and the substituents attached to the phosphorus atoms influence the chemical reactivity of the diphosphine ligand in metal complexes through steric and electroniceffects ${ }^{13}$.Phosphorus ligands typically bind to the metal centers via the lone pair of electrons on the phosphorus ${ }^{14}$.

\section{EXPERIMENTAL}

\section{Materials and Instrumentation}

The compounds $\mathrm{PtCl}_{2}, \mathrm{PdCl}_{2}$, dppm, dppp, dppf and $\mathrm{S}-1 \mathrm{H}$ benzo[d] imidazole-2-yl benzothioate (HSBIBT) were commerciallyavailable and obtained from Yacoo chem. China. The complexes $\left[\mathrm{Pd}\left(\mathrm{k}^{2}-\mathrm{dppf}\right)\right.$ (HSBIBT)Cl]Cl, [Pd(k $\left.{ }^{2}-d p p p\right)$ (HSBIBT)Cl]Cl, [Pt(k ${ }^{2}-$ $\mathrm{dppm}$ ) (HSBIBT)Cl]Cl were prepared according to the literature.

Elemental CHNS analysis were carried out on a EuroEA 3000 instrument. The FT-IR spectra in range (4000-200 $\mathrm{cm}^{-1}$ ) were recorded as CsI disc on Shimadzu IRAffinity-1S FTIR Spectrophotometer, and FT-IR spectra in range $\left(4000-400 \mathrm{~cm}^{-1}\right)$ were recorded as $\mathrm{KBr}$ disc on Shimadzu, FT-IR spectroscopy Mod IR Affinity-1CE spectrophotometer. UV-Visible spectra were measured using AE-UV1609 (UK) CO., LTD Shimadzu, in the range $(200-800) \mathrm{nm}$. The magnetic susceptibility values of the prepared complexes were carried out at room temperature using Bruker Magnet BM6. Conductivity measurements were made on a conductivity meter type Senz $\mu$ Siemen tester 4200 (093 cell constant) (UK.). Melting points were measured on Melting Point-MPD-100 Pixel Technology CO., Limited apparatus.

\section{Synthesis of $\left[\mathrm{Pd}\left(\mathbf{k}^{2}-\mathrm{dppf}\right) \mathrm{Cl}_{2}\right] \cdot \mathrm{CH}_{2} \mathrm{Cl}_{2}$ (1)}

The solution of dppf $(1.6632 \mathrm{~g}, 3 \mathrm{mmol})$ in $15 \mathrm{ml} \mathrm{CH}_{2} \mathrm{Cl}_{2}$ was added to a solution of $\mathrm{PdCl}_{2}$ $(0.5320 \mathrm{~g}, 3 \mathrm{mmol})$ that dissolved in $2 \mathrm{~cm}^{3}$ ethanol and $2 \mathrm{~cm}^{3}$ concentrate $\mathrm{HCl}$. The mixture was reflux for $3 \mathrm{hrs}$. And then the solvent was evaporate at room temperature to give red precipitate. A small portion of this complex was dissolve in $\mathrm{CH}_{2} \mathrm{Cl}_{2}$ and set to slow evaporation at room temperature after a few days, the red colored needle crystals were formed suitable for single-crystal diffraction analysis ${ }^{15}$. The melting point is $253^{\circ} \mathrm{C}$, yield is $2.086 \mathrm{gm}(95 \%)$, formula: $\mathrm{Pd}\left(\mathrm{k}^{2}-\right.$ dppf) $\mathrm{Cl}_{2}$ ] and M.wt.is $816.58 \mathrm{gm} / \mathrm{mole}$, as shown in Figure 1.

\section{Synthesis of $\left[\mathrm{Pd}\left(\mathbf{k}^{2}\right.\right.$-dppf)(HSBIBT)Cl] $\mathrm{Cl}(2)$}

A solution of ligand $(0.0509 \mathrm{~g}, 0.2 \mathrm{mmol})$ in $\mathrm{CH}_{2} \mathrm{Cl}_{2}\left(15 \mathrm{~cm}^{3}\right)$ was added to a solution of $\left[\mathrm{Pd}\left(\mathrm{k}^{2}\right.\right.$-dppf $\left.) \mathrm{Cl}_{2}\right](0.1463 \mathrm{~g}, 0.2 \mathrm{mmol})$ in $\mathrm{CH}_{2} \mathrm{Cl}_{2}$ $\left(15 \mathrm{~cm}^{3}\right)$, and the mixture was heated under reflux for $4 \mathrm{hrs}$ and then the Solvent was evaporated at room temperature to give a red solid. Yield $=91 \%$, m. $p=139-141^{\circ} \mathrm{C}$. as shown in Figure 1.

\section{Synthesis of $\left[\mathrm{Pd}\left(\mathrm{k}^{2}-\mathrm{dppp}\right) \mathrm{Cl}_{2}\right]$ (3)}

The solution of dppp $(1.2373 \mathrm{~g}, 3 \mathrm{mmol})$ in $15 \mathrm{ml} \mathrm{CH}_{2} \mathrm{Cl}_{2}$ was added to a solution of $\mathrm{PdCl}_{2}$ $(0.5320 \mathrm{~g}, 3 \mathrm{mmol})$ that dissolved in $2 \mathrm{~cm}^{3}$ ethanol and $2 \mathrm{~cm}^{3}$ concentrate $\mathrm{HCl}$. The mixture was reflux for $3 \mathrm{hrs}$. And then the solvent was evaporate at room temperature to give pale yellow precipitate. A small portion of this complex was dissolve in $\mathrm{CH}_{2} \mathrm{Cl}_{2}$ and set to slow evaporation at room temperature after a one week, the yellow colored needle crystals were formed suitable for single-crystal diffraction analysis ${ }^{15}$. The Melting point is $277^{\circ} \mathrm{C}$, yield is $1.664 \mathrm{gm}(94 \%)$, formula: $\left[\mathrm{Pd}\left(\mathrm{k}^{2}-\mathrm{dppp}\right) \mathrm{Cl}_{2}\right]$ and M.wt.is 589.72 $\mathrm{gm} / \mathrm{mole}$.as shown in Figure 1.

\section{Synthesis of $\left[\mathrm{Pd}\left(\mathrm{k}^{2}\right.\right.$-dppp)(HSBIBT)Cl]Cl(4)}

A solution of ligand $(0.0509 \mathrm{~g}, 0.2 \mathrm{mmol})$ in $\mathrm{CH}_{2} \mathrm{Cl}_{2}\left(15 \mathrm{~cm}^{3}\right)$ was added to a solution of $\left[\mathrm{Pd}\left(\mathrm{k}^{2}-\right.\right.$ 
dppp) $\left.\mathrm{Cl}_{2}\right](0.1179 \mathrm{~g}, 0.2 \mathrm{mmol})$ in $\mathrm{CH}_{2} \mathrm{Cl}_{2}\left(15 \mathrm{~cm}^{3}\right)$, and the mixture was heated under reflux for $4 \mathrm{hrs}$ and then the Solvent was evaporated at room temperature to give a yellow solid. Yield $=93 \%$, m. $p=142-144^{\circ} \mathrm{C}$. as shown in Figure 1.

\section{Synthesis of $\left[\mathrm{Pt}\left(\mathrm{k}^{2}-\mathrm{dppmCl}\right) \mathrm{Cl} 2\right](5)$}

The solution of dppm (1.1532g, 3mmol) in $15 \mathrm{ml} \mathrm{CH} \mathrm{Cl}_{2}$ was added to a solution of $\mathrm{PtCl}_{2}$ $(0.7979 \mathrm{~g}, 3 \mathrm{mmol})$ that dissolved in $2 \mathrm{~cm}^{3}$ ethanol and $2 \mathrm{~cm}^{3}$ concentrate $\mathrm{HCl}$. The mixture was reflux for $3 \mathrm{hrs}$. And then the solvent was evaporate at room temperature to give yellow precipitate. A small portion of this complex was dissolve in $\mathrm{CH}_{2} \mathrm{Cl}_{2}$ and set to slow evaporation at room temperature after a one week, the yellow colored needle crystals were formed suitable for single-crystal diffraction analysis ${ }^{15}$. The melting point is $>300^{\circ} \mathrm{C}$, yield is 1.756gm (90\%), formula: $\left[\mathrm{Pt}\left(\mathrm{k}^{2}-\mathrm{dppmCl}\right) \mathrm{Cl}_{2}\right]$ and M.wt.is $667.43 \mathrm{gm} / \mathrm{mole}$.as shown in Figure 1.

\section{Synthesis of [Pt( $\mathrm{k}^{2}$-dppmCl)(HSBIBT)Cl] Cl(6)}

A solution of ligand $(0.0509 \mathrm{~g}, 0.2 \mathrm{mmol})$ in $\mathrm{CH}_{2} \mathrm{Cl}_{2}\left(15 \mathrm{~cm}^{3}\right)$ was added to a solution of $\left[\mathrm{Pt}\left(\mathrm{k}^{2}\right.\right.$-dppmCl$\left.) \mathrm{Cl}_{2}\right](0.1300 \mathrm{~g}, 0.2 \mathrm{mmol})$ in $\mathrm{CH}_{2} \mathrm{Cl}_{2}$ $\left(15 \mathrm{~cm}^{3}\right)$, and the mixture was heated under reflux for $4 \mathrm{hrs}$ and then the Solvent was evaporated at room temperature to give a yellow solid. Yield $=90 \%$, $\mathrm{m} . \mathrm{p}=117-119^{\circ} \mathrm{C}$. as shown in Figure 1.

\section{X-ray crystallography}

Single crystal X-ray crystallography studies were performed at $200 \mathrm{k}$ using a Bruker Kappa Apex II diffractometer with graphite monochromated Mo $K \alpha$ radiation $\left(\lambda=0.71073^{\circ} \mathrm{A}\right)$. For data collection, Apex-II was used while for cell refinement and data reduction, SAINT was used ${ }^{16}$. The structures were solved by direct methods applying SHELXL-2013 ${ }^{17}$, with SHELXLE ${ }^{18}$ as a graphical interface.

Red crystals of 1 , yellow crystals of 3 and yellow crystals of 5 suitable for $\mathrm{X}$-ray crystallographic measurements were obtained by slow evaporation of dichloromethane solutions of the respective complex. Table 4 gives the crystallographic data and collection parameters. All Non-hydrogen atoms were refined with anisotropic displacement parameters. Hydrogen atoms were included in the models by calculating the positions (riding model) and refined with calculated isotropic displacement parameters.

\section{RESULTS and DISCUSSIONS}

The mixed ligand complexes were synthesized by two steps. The first step include the preparation of metal complexes with tertiary diphosphines ligands $\left[\mathrm{MCl}_{2}\left(\mathrm{k}^{2}-\mathrm{PPh}_{2}\left(\mathrm{CH}_{2}\right) n P P h_{2}\right)\right]$ where $n=1,3$ with $\left[\mathrm{MCl}_{2}\left(\mathrm{k}^{2}-\mathrm{dppf}\right)\right]$, and $\mathrm{M}=\mathrm{Pd}$ and $\mathrm{Pt}$. The second step include the synthesis of mixed ligand complexes by addition of $\mathrm{S}-1 \mathrm{H}$-benzo[d] imidazole-2-yl benzothioate (HSBIBT) ligand to tertiary diphosphines metal complexes. The physical data of complexes are given in Table (1), the suggested molecular formula are supported by IR spectra, UV-Visible sepectra, elemental analysis, magnetic moment and conductivity measurement.

\section{IR spectral studies}

The infraredspectrumoffree (HSBIBT) ligand ischaracterized by intense absorption bandat $1712 \mathrm{~cm}^{-1}$. This band is assigned to $v(\mathrm{C}=\mathrm{O})$ group ${ }^{19,4}$. The stretching frequency of $v(\mathrm{C}=\mathrm{N})$ bandwhich appeared at $1618 \mathrm{~cm}^{-1}$ forfree (HSBIBT) ligand. This band was shifted to lower frequency during

Table 1: physical characteristics and analytical data for metal complexes

\begin{tabular}{|c|c|c|c|c|c|c|c|}
\hline \multirow[t]{2}{*}{ Complex } & \multirow[t]{2}{*}{ Color } & \multirow[t]{2}{*}{ Yield \% } & \multirow[t]{2}{*}{ M.P ${ }^{\circ} \mathbf{C}$} & \multicolumn{4}{|c|}{ Elemental analysis, calc. (found) $\%$} \\
\hline & & & & C & $\mathbf{H}$ & $\mathbf{N}$ & $\mathrm{S}$ \\
\hline [Pd(k²-dppf)(HSBIBT) & Red & 91 & $142-144$ & 58.47 & 3.84 & 2.75 & 3.15 \\
\hline $\mathrm{Cl}] \mathrm{Cl}$ & & & & -58.47 & -3.75 & -2.84 & -3.24 \\
\hline$\left[\mathrm{Pd}\left(\mathrm{k}^{2}-\mathrm{dppp}\right)(\mathrm{HSBIBT})\right.$ & Yellow & 93 & $139-141$ & 58.45 & 4.18 & 3.26 & 3.46 \\
\hline $\mathrm{Cl}] \mathrm{Cl}$ & & & & -58.35 & -4.15 & -3.32 & -3.79 \\
\hline$\left[\mathrm{Pt}\left(\mathrm{k}^{2}-\mathrm{dppmCl}\right)\right.$ & Yellow & 90 & $117-119$ & 51.46 & 3.6 & 2.93 & 3.44 \\
\hline (HSBIBT)Cl]Cl & & & & -51.78 & -3.43 & -3.09 & -3.54 \\
\hline
\end{tabular}


complexationdue to the coordination of nitrogen atom to the metals ${ }^{20}$ (Table 2).

The infrared spectra of complexes showed medium intensity bands in the range 513 to $570 \mathrm{~cm}^{-1}$, these bands are attributed to $v(\mathrm{M}-\mathrm{N})^{21}$. Also showed a bands in the range 370 to $391 \mathrm{~cm}^{-1}$, these bands are related tov $(\mathrm{M}-\mathrm{Cl})^{22}$. Further support for the coordinated phosphines ligands has been provided by the appearance of new bands in the range 285 to $291 \mathrm{~cm}^{-1}$, these bands are assigned toí(M-P) ${ }^{23}$.

\section{Magnetic moments}

The magnetic moments of complexes [Pd(k $\mathrm{k}^{2}$-dppf)(HSBIBT)Cl]Cl (2), [Pd(k $\mathrm{k}^{2}$-dppp)(HSBIBT) $\mathrm{Cl} \mathrm{Cl}(4)$ and $\left[\mathrm{Pt}\left(\mathrm{k}^{2}-\mathrm{dppmCl}\right)(\mathrm{HSBIBT}) \mathrm{Cl}\right] \mathrm{Cl}$ (6) are $0.54,0.6$ and 0.44 B.M. respectively (Table 3), suggesting a square planer arrangement around the metal and complexes are diamagnetic ${ }^{24,25}$.

\section{Electronic spectral studies}

The UV-Vis spectrum in $10^{-3} \mathrm{M}$ chloroform (Table 3)shows three absorption bands of $\mathrm{Pd}(\mathrm{II})$ complex (2), at $20661 \mathrm{~cm}^{-1}, 28571 \mathrm{~cm}^{-1}$, and $33783 \mathrm{~cm}^{-1}$ which are assigned to the ${ }^{1} \mathrm{~A}_{1} \mathrm{~g} \rightarrow{ }^{1} \mathrm{~A}_{2} \mathrm{~g}$, ${ }^{1} A_{1} g \rightarrow{ }^{1} \mathrm{Eg}$ and charge transfer transition respectively, these bands are reasonable to square planner geometry ${ }^{26}$. The UV-visible spectrum of $\mathrm{Pd}(\mathrm{II})$ complex(4), gave three spins allowed transitions at $24038 \mathrm{~cm}^{-1}, 29411 \mathrm{~cm}^{-1}$, and $32679 \mathrm{~cm}^{-1}$ which are assigned to the ${ }^{1} \mathrm{~A}_{1} g \rightarrow{ }^{1} \mathrm{~A}_{2} g,{ }^{1} \mathrm{~A}_{1} g \rightarrow{ }^{1} \mathrm{Eg}$ and charge transfer transition respectively. The UVvisible spectrum of $\mathrm{Pt}(\mathrm{II})$ complex(6), shows three absorption bands at $23923 \mathrm{~cm}^{-1}, 27777 \mathrm{~cm}^{-1}$, and $32573 \mathrm{~cm}^{-1}$ which are assigned to the ${ }^{1} A_{1} g \rightarrow{ }^{1} A_{2} g$, ${ }^{1} A_{1} g \rightarrow{ }^{1} \mathrm{Eg}$ and charge transfer transition respectively, These transitions value are indicated to square planner geometry ${ }^{27}$.

\section{Conductivity measurement}

The molar conductivity of complexes $\left[\mathrm{Pd}\left(\mathrm{k}^{2}-\right.\right.$ dppf)(HSBIBT)Cl]Cl (2), [Pd(k $\left.{ }^{2}-d p p p\right)(H S B I B T)$ $\mathrm{Cl}] \mathrm{Cl}(4)$ and $\left[\mathrm{Pt}\left(\mathrm{k}^{2}-\mathrm{dppmCl}\right)(\mathrm{HSBIBT}) \mathrm{Cl}\right] \mathrm{Cl}$ (6) in dimethyl sulfoxide (DMSO) are 34.2, 41.4 and $36\left(\mathrm{~cm}^{2} . \mathrm{ohm}^{-1} \cdot \mathrm{mol}^{-1}\right)$ (Table 3)suggesting that they are electrolytescomplexes ${ }^{28,29}$.

\section{X-ray Crystal structures}

The $\left[\mathrm{Pd}\left(\mathrm{k}^{2}\right.\right.$-dppf $\left.) \mathrm{Cl}_{2}\right] \cdot \mathrm{CH}_{2} \mathrm{Cl}_{2}$ complex was crystallized in a dichorometahne $\left(\mathrm{CH}_{2} \mathrm{Cl}_{2}\right)$ at room temperature. After one week, the red colored needle type crystals had grown from the $\mathrm{CH}_{2} \mathrm{Cl}_{2}$ solvent

Table 2: IR frequencies $\left(\mathrm{cm}^{-1}\right)$ of the compounds

\begin{tabular}{lcccccccc}
\hline Complex & $v(\mathbf{N}-\mathbf{H})$ & $v(\mathbf{C}=\mathbf{O})$ & $v(\mathbf{C}=\mathbf{N})$ & $v(\mathbf{P}-\mathbf{P h})$ & $v(\mathbf{P}-\mathbf{C})$ & $v(\mathbf{M}-\mathbf{N})$ & $v(\mathbf{M}-\mathbf{P})$ & $v(\mathbf{M}-\mathbf{C l})$ \\
\hline HSBIBT & 3151 & 1712 & 1618 & - & - & - & - & - \\
2 & 3165 & 1712 & 1608 & 1435 & - & 513 & 285 & 370 \\
4 & 3159 & 1712 & 1612 & 1435 & 513 & 540 & 288 & 383 \\
6 & 3153 & 1712 & 1608 & 1436 & 509 & 559 & 291 & 391 \\
\hline
\end{tabular}

Table 3: Electronic spectra, magnetic moments and molar conductivity of complexes

\begin{tabular}{|c|c|c|c|c|}
\hline complex & $\begin{array}{l}\lambda \max (\mathrm{nm}) / \\
\text { Chloroform }\end{array}$ & assignments & $\mu_{\text {eff }}(B M)^{a}$ & $\begin{array}{c}\Lambda_{\mathrm{M}}\left(\Omega_{-1} \mathrm{~cm}^{2} \mathrm{~mol}^{-1}\right) / \\
\text { DMSO }\end{array}$ \\
\hline 2 & $\begin{array}{l}484 \\
350 \\
296\end{array}$ & $\begin{array}{l}{ }^{1} A_{19} \rightarrow{ }^{1} A_{2 g} \\
{ }^{1} A_{19} \rightarrow{ }^{1} E_{g} \\
\text { C.T }\end{array}$ & 0.54 & 34.2 \\
\hline 4 & $\begin{array}{l}416 \\
340 \\
306\end{array}$ & $\begin{array}{l}{ }^{1} A_{1 g} \rightarrow{ }^{1} B_{19} \\
1 A_{1 g} g \rightarrow 1 \mathrm{Eg} \\
\text { C.T }\end{array}$ & 0.6 & 41.4 \\
\hline 6 & $\begin{array}{l}418 \\
360 \\
307\end{array}$ & $\begin{array}{l}{ }^{1} A_{1 g} \rightarrow{ }^{1} A_{2 g} \\
{ }^{1} A_{1 g} \rightarrow{ }^{1} E_{g} \\
\text { C. } \mathrm{T}\end{array}$ & 0.44 & 36 \\
\hline
\end{tabular}


Table 4: Crystallographic Data and structure refinementfor $\left[\mathrm{Pd}\left(k^{2}-\mathrm{dppf}\right) \mathrm{Cl}_{2}\right] \cdot \mathrm{CH}_{2} \mathrm{Cl}_{2}$, $\left[\mathrm{Pd}\left(\mathrm{k}^{2}-\mathrm{dppp}\right) \mathrm{Cl}_{2}\right]$ and $\left[\mathrm{Pt}\left(\mathrm{k}^{2}\right.\right.$-dppmCl$\left.) \mathrm{Cl}_{2}\right]$ complexes.

\begin{tabular}{|c|c|c|c|}
\hline & $\begin{array}{l}{\left[\mathrm{Pd}\left(\mathrm{K}^{2}-\mathrm{dppf}\right) \mathrm{Cl}_{2}\right] .} \\
\mathrm{CH}_{2} \mathrm{Cl}_{2}\end{array}$ & {$\left[\mathrm{Pd}\left(\mathrm{k}^{2}\right.\right.$-dppp $\left.) \mathrm{Cl}_{2}\right]$} & {$\left[\mathrm{Pt}\left(\mathrm{k}^{2}-\mathrm{dppmCl}\right) \mathrm{Cl}_{2}\right]$} \\
\hline Empirical formula & $\begin{array}{l}\mathrm{C}_{34} \mathrm{H}_{28} \mathrm{Cl}_{2} \mathrm{FeP}_{2} \\
\text { Pd. } \mathrm{CH}_{2} \mathrm{Cl}_{2}\end{array}$ & $\mathrm{C}_{27} \mathrm{H}_{26} \mathrm{Cl}_{2} \mathrm{P}_{2} \mathrm{Pd}$ & $\begin{array}{l}\mathrm{C}_{24 \cdot 27} \mathrm{H}_{21}{ }_{246} \mathrm{Cl}_{2} \mathrm{P}_{2} \\
\mathrm{Pt}(\mathrm{Cl})_{0.729}(\mathrm{H})_{0.54}\end{array}$ \\
\hline Formula weight & 816.58 & 589.72 & 667.43 \\
\hline $\mathrm{R}$ & 0.03 & 0.02 & 0.03 \\
\hline Temperature & $200 \mathrm{~K}$ & $200 \mathrm{~K}$ & $200 \mathrm{k}$ \\
\hline Radiation & $0.71073 \mathrm{~A}$ & $0.71073 \mathrm{~A}$ & $0.71073 \mathrm{~A}$ \\
\hline Crstal system & Monoclinic & triclinic & Monoclinic \\
\hline space group & $\mathrm{P} 21 / \mathrm{c}$ & $\mathrm{P}-1$ & $\mathrm{C} 2 / \mathrm{c}$ \\
\hline $\mathrm{a}\left({ }^{\circ} \mathrm{A}\right)$ & $9.7960(3)$ & $8.4865(3)$ & $16.2034(5)$ \\
\hline $\mathrm{b}\left({ }^{\circ} \mathrm{A}\right)$ & $18.1527(7)$ & $10.5484(4)$ & $7.8274(2)$ \\
\hline$c\left({ }^{\circ} A\right)$ & $19.0713(7)$ & $14.4020(6)$ & $19.2496(6)$ \\
\hline '(deg) & 90 & $88.388(2)$ & 90 \\
\hline${ }^{2}(\mathrm{deg})$ & $102.536(1)$ & $80.064(2)$ & $98.918(1)$ \\
\hline${ }^{3}(\mathrm{deg})$ & 90 & $73.475(2)$ & 90 \\
\hline volume & $3310.5(2) A^{\wedge} 3$ & $1217.16(8) \mathrm{A}^{\wedge} 3$ & $2411.92(12) \mathrm{A}^{\wedge 3}$ \\
\hline$F(000)$ & 1640 & 596 & $4,1.838 \mathrm{Mg} / \mathrm{m}^{\wedge} 3$ \\
\hline Z, Calculated density & $4,1.638 \mathrm{Mg} / \mathrm{m}^{\wedge} 3$ & $2,1.609 \mathrm{~g} / \mathrm{m}^{\wedge} 3$ & $6.263 \mathrm{~mm}^{\wedge}-1$ \\
\hline Absorption coefficient & $1.426 \mathrm{~mm}^{\wedge}-1$ & $1.128 \mathrm{~mm}^{\wedge}-1$ & 1288 \\
\hline Crystal size & $0.41 \times 0.46 \times 0.47 \mathrm{~mm}$ & $\begin{array}{l}0.19 \times 0.31 \\
\times 0.51 \mathrm{~mm}\end{array}$ & $\begin{array}{l}0.04 \times 0.20 \\
\times 0.21 \mathrm{~mm}\end{array}$ \\
\hline Dataset & $-13: 13 ;-20: 24 ;-25: 25$ & $\begin{array}{l}-11: 11 ;-13: 14 ; \\
-18: 19\end{array}$ & $\begin{array}{l}-13: 21 ;-10: 10 \\
-25: 25\end{array}$ \\
\hline $\begin{array}{l}\text { Reflections collected / } \\
\text { unique }\end{array}$ & $\begin{array}{l}60083,8203 \\
{[R(\text { int })=0.018]}\end{array}$ & $\begin{array}{l}32265,6016 \\
{[R(\text { int })=0.016]}\end{array}$ & $\begin{array}{l}16294,3007 \\
{[R(\text { int })=0.030]}\end{array}$ \\
\hline $\begin{array}{l}\text { Observed Data } \\
{[\mathrm{I}>2.0 \text { sigma(I)] }}\end{array}$ & 7414 & 5668 & 2930 \\
\hline $\mathrm{R}, \mathrm{wR} 2, \mathrm{~S}$ & $\begin{array}{l}R=0.0284, w R 2=0.0693 \\
S=1.04\end{array}$ & $\begin{array}{l}R=0.0177 \\
w R 2=0.0451, S=1.03\end{array}$ & $\begin{array}{l}\mathrm{R}=0.0478, \\
\mathrm{wR} 2=0.1178, S=1.30\end{array}$ \\
\hline Min. and Max. Resd. Dens & {$[-2.08,1.73] \mathrm{e} / \mathrm{Ang}^{\wedge} 3$} & {$[-0.44,0.44] \mathrm{e} / \mathrm{Ang}{ }^{\wedge 3}$} & {$[-1.71,4.83] \mathrm{e} / \mathrm{Ang}{ }^{\wedge} 3$} \\
\hline Max. and Av & 0.00 and 0.00 & 0.00 and 0.00 & 0.00 and 0.00 \\
\hline
\end{tabular}

Table 5: Selected bond distances (in $\mathrm{A}^{\circ}$ ) and bond angles (in degree) for $\left[\mathrm{Pd}\left(\mathrm{k}^{2}-\mathrm{dppf}\right) \mathrm{Cl}_{2}\right]$. $\mathrm{CH}_{2} \mathrm{Cl}_{2}$

Bond distance

Bond angle

$\mathrm{Pd}(1)-\mathrm{Cl}(1) \quad 2.3417(7) \quad \mathrm{Cl}(1)-\mathrm{Pd}(1)-\mathrm{P}(1) \quad 87.79(2)$

$\mathrm{Pd}(1)-\mathrm{Cl}(2) \quad 2.3577(7) \quad \mathrm{Cl}(1)-\mathrm{Pd}(1)-\mathrm{P}(2) \quad 171.50(3)$

$\mathrm{Pd}(1)-\mathrm{P}(1) \quad 2.2774(7) \quad \mathrm{Cl}(2)-\mathrm{Pd}(1)-\mathrm{P}(1) \quad 177.75(2)$

$\mathrm{Pd}(1)-\mathrm{P}(2) \quad 2.2883(7) \quad \mathrm{Cl}(2)-\mathrm{Pd}(1)-\mathrm{P}(2) \quad 83.96(2)$

$\mathrm{Fe}(1)-\mathrm{C} 1(1) \quad 1.996(2) \quad \mathrm{P}(1)-\mathrm{Pd}(1)-\mathrm{P}(2) \quad$ 97.94(2)

$\mathrm{Fe}(1)-\mathrm{C} 1(2) \quad 2.028(2) \quad \mathrm{C} 1(3)-\mathrm{Fe}-\mathrm{C} 1(4) \quad 40.16(10)$

$\mathrm{P}(1)-\mathrm{C} 1(1) \quad 1.802(2) \quad \mathrm{Pd}(1)-\mathrm{P}(1)-\mathrm{C}(31)$ 108.46(7) and these crystals were subjected to single-crystal $X$-raydiffraction study. The molecular structure of $\left[\mathrm{Pd}\left(\mathrm{k}^{2}-\mathrm{dppf}\right) \mathrm{Cl}_{2}\right] \cdot \mathrm{CH}_{2} \mathrm{Cl}_{2}$ complex is shown in Figure 1. The $\left[\mathrm{Pd}\left(\mathrm{k}^{2}-\mathrm{dppf}\right) \mathrm{Cl}_{2}\right] \cdot \mathrm{CH}_{2} \mathrm{Cl}_{2}$ complex crystallizes in a monoclinic space group Monoclinic, $\mathrm{P} 21 / \mathrm{c} . \mathrm{a}=9.7960(3) \mathrm{A}, \mathrm{b}=18.1527(7) \mathrm{A}, \mathrm{c}=19.0713(7) \mathrm{A}$, $\alpha=\gamma=90^{\circ}, \beta=102.536(1)$. Structural analysis reveals that $\left[\mathrm{Pd}\left(\mathrm{k}^{2}\right.\right.$-dppf $\left.) \mathrm{Cl}_{2}\right] \cdot \mathrm{CH}_{2} \mathrm{Cl}_{2}$ complex has a square planer geometry around the metal center, two chlorines and two phosphorus atoms are coordinated with the palladium ion. The dppf behave as bidentate chelating ligand. The crystal determination data and selected bond distances and bond angles for 


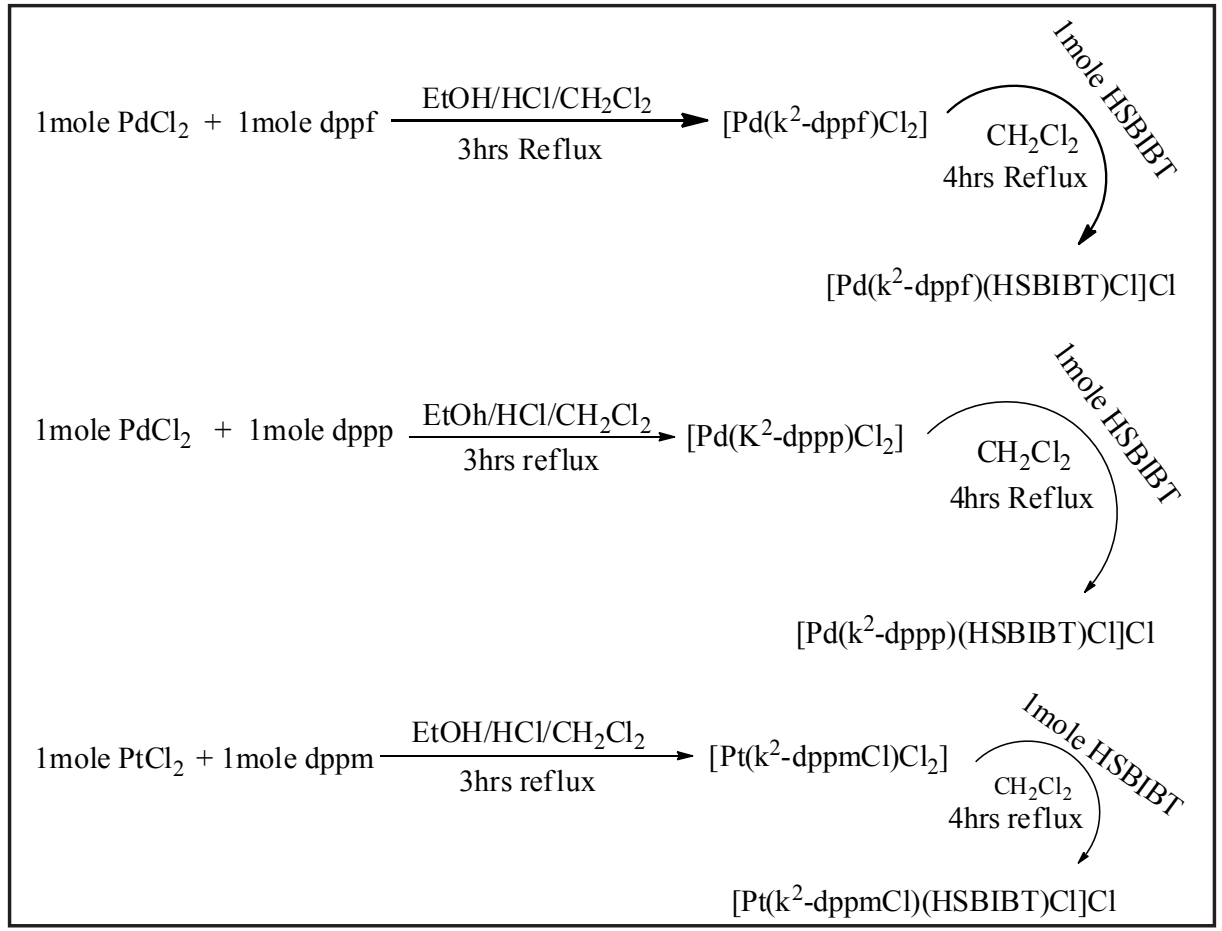

Table 6: Selected bond distances (in $\mathrm{A}^{\circ}$ ) and bond angles (in degree) for $\left[\mathrm{Pd}\left(\mathrm{k}^{2}-\mathrm{dppp}\right) \mathrm{Cl}_{2}\right]$

\begin{tabular}{llll}
\hline \multicolumn{2}{c}{ Bond distance } & \multicolumn{2}{c}{ Bond angle } \\
\hline $\mathrm{Pd}(1)-\mathrm{Cl}(1)$ & $2.3575(4)$ & $\mathrm{Cl}(1)-\mathrm{Pd}(1)-\mathrm{Cl}(2)$ & $90.54(1)$ \\
$\mathrm{Pd}(1)-\mathrm{Cl}(2)$ & $2.3557(4)$ & $\mathrm{Cl}(1)-\mathrm{Pd}(1)-\mathrm{P}(1)$ & $87.77(1)$ \\
$\mathrm{Pd}(1)-\mathrm{P}(1)$ & $2.2424(4)$ & $\mathrm{Cl}(1)-\mathrm{Pd}(1)-\mathrm{P}(2)$ & $177.80(2)$ \\
$\mathrm{Pd}(1)-\mathrm{P}(2)$ & $2.2456(4)$ & $\mathrm{Cl}(2)-\mathrm{Pd}(1)-\mathrm{P}(1)$ & $172.11(2)$ \\
$\mathrm{P}(1)-\mathrm{C}(1)$ & $1.8243(16)$ & $\mathrm{Cl}(2)-\mathrm{Pd}(1)-\mathrm{P}(2)$ & $91.39(1)$ \\
$\mathrm{P}(2)-\mathrm{C}(3)$ & $1.8355(16)$ & $\mathrm{P}(1)-\mathrm{Pd}(1)-\mathrm{P}(2)$ & $90.47(1)$ \\
$\mathrm{C}(3)-\mathrm{H}(3) \mathrm{A}$ & 0.9900 & $\mathrm{Pd}(1)-\mathrm{P}(1)-\mathrm{C}(1)$ & $116.42(5)$ \\
\hline
\end{tabular}

Table 7: Selected bond distances (in $\mathrm{A}^{\circ}$ ) and bond angles (in degree) for $\left[\mathrm{Pt}\left(\mathrm{k}^{2}-\mathrm{dppmCl}\right) \mathrm{Cl}_{2}\right]$

Bond distance

\begin{tabular}{llll}
$\mathrm{Pt}(1)-\mathrm{Cl}(1)$ & $2.3661(19)$ & $\mathrm{Cl}(1)-\mathrm{Pt}(1)-\mathrm{P}(2)$ & $98.10(7)$ \\
$\mathrm{Pt}(1)-\mathrm{P}(2)$ & $2.217(2)$ & $\mathrm{Cl}(1)-\mathrm{Pt}(1)-\mathrm{P}(1)$ & $98.10(7)$ \\
$\mathrm{Pt}(1)-\mathrm{P}(1)$ & $2.217(2)$ & $\mathrm{Cl}(1)-\mathrm{Pt}(1)-\mathrm{Cl}(1) \_\mathrm{a}$ & $90.02(6)$ \\
$\mathrm{Pt}(1)-\mathrm{Cl}(1) \_\mathrm{a}$ & $2.3661(19)$ & $\mathrm{Cl}(1)-\mathrm{Pt}(1)-\mathrm{P}(2) \_\mathrm{a}$ & $171.58(7)$ \\
$\mathrm{Pt}(1)-\mathrm{P}(2) \_\mathrm{a}$ & $2.217(2)$ & $\mathrm{Cl}(1) \_\mathrm{a}-\mathrm{Pt}(1)-\mathrm{P}(2)$ & $171.58(7)$ \\
$\mathrm{P}(1)-\mathrm{C}(21)$ & $1.816(7)$ & $\mathrm{P}(2)-\mathrm{Pt}(1)-\mathrm{P}(2) \_\mathrm{a}$ & $73.87(8)$ \\
$\mathrm{P}(2)-\mathrm{C}(11)$ & $1.808(8)$ & $\mathrm{Pt}(1)-\mathrm{P}(1)-\mathrm{C}(21)$ & $117.2(3)$ \\
\hline
\end{tabular}




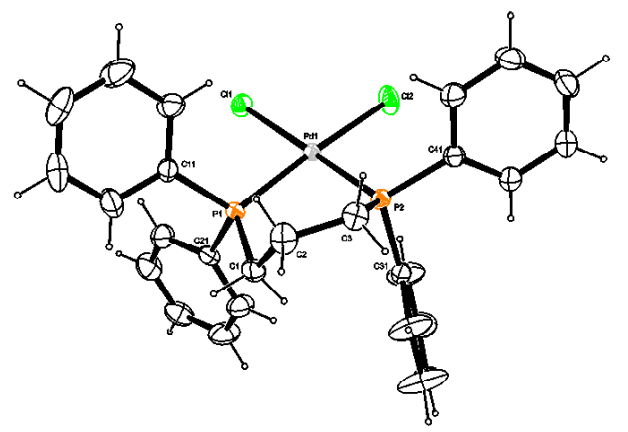

Fig. 1: synthesis route of complexes

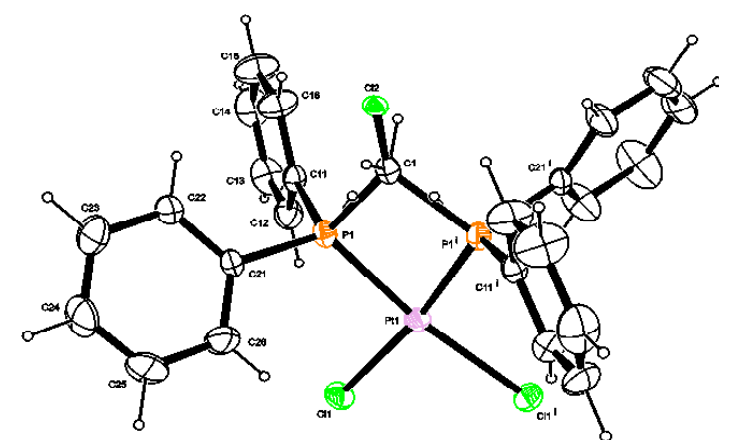

Fig. 3: Molecular Structure of $\left[\mathrm{Pd}\left(\mathrm{k}^{2}-\mathrm{dppp}\right) \mathrm{Cl}_{2}\right]$ Complex

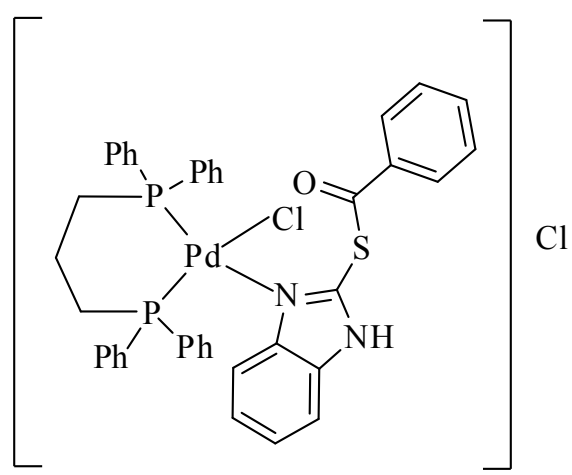

Fig. 5: The Proposed Geometrical Structure of [Pd(k²-dppp)(HSBIBT)Cl]CIComplex

$\left[\mathrm{Pd}\left(\mathrm{k}^{2}\right.\right.$-dppf $\left.) \mathrm{Cl}_{2}\right] \cdot \mathrm{CH}_{2} \mathrm{Cl}_{2}$ complex are summarized in Tables 4and5. The palladium center was found to a distorted square planar geometry, with Cl1-Pd1-P1, $\mathrm{Cl} 2-\mathrm{Pd} 1-\mathrm{P} 2, \mathrm{Cl} 1-\mathrm{Pd} 1-\mathrm{P} 2$ and $\mathrm{Cl} 2-\mathrm{Pd} 1-\mathrm{P} 1$ angles of $87.79(2), 83.96(2), 171.50(3)$, and 177.75(2) respectively ${ }^{30,31}$.

The $\left[\mathrm{Pd}\left(\mathrm{k}^{2}-\mathrm{dppp}\right) \mathrm{Cl}_{2}\right]$ complex was crystallized from $\mathrm{C} \mathrm{CH}_{2} \mathrm{Cl}_{2}$ solution of the complex at

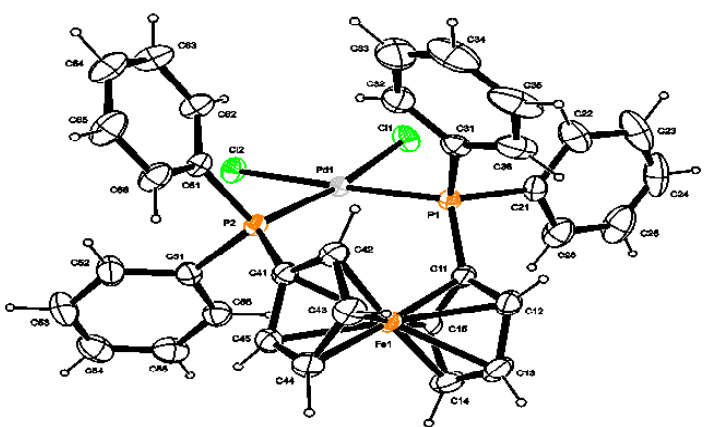

Fig. 2: Molecular Structure of $\left[\mathrm{Pd}\left(\mathbf{k}^{2}-\mathrm{dppf}\right) \mathrm{Cl}_{2}\right]$. $\mathrm{CH}_{2} \mathrm{Cl}_{2}$ Complex

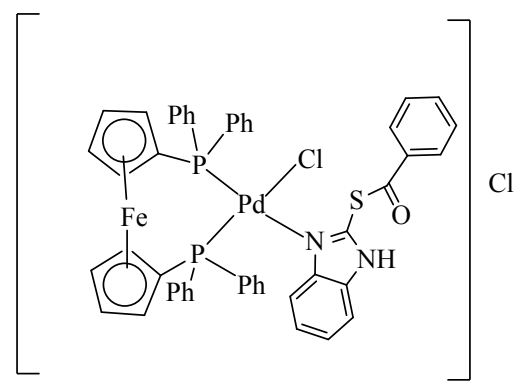

Fig. 4: The Proposed Geometrical Structure of [Pd( $k^{2}$-dppf)(HSBIBT)Cl]Cl Complex<smiles></smiles>

Fig. 6: The Proposed Geometrical Structure of [Pt(k $k^{2}$-dppmCl)(HSBIBT)Cl]ClComplex

room temperature, after a one week, as yellow colored needle type crystals are formed, these crystals were subjected to single-crystal X-ray diffractometer study. The molecular structure of $\left[\mathrm{Pd}\left(\mathrm{k}^{2}-\mathrm{dppp}\right) \mathrm{Cl}_{2}\right]$ complex is shown in Fig. 2. The structural analysis reveals that $\left[\mathrm{Pd}\left(\mathrm{k}^{2}-\mathrm{dppp}\right) \mathrm{Cl}_{2}\right]$ complex has a distorted square planer geometry around the metal center and that the two phosphorus and two chlorine atoms are coordinated to the palladium ion. The dppm behave 
as behave as bidentate chelating ligand. Whereas the Pd-P bond distance of 2.2424(4) and 2.2456(4) $\AA$ for $\mathrm{P}(1)$ and $\mathrm{P}(2)$ atoms of the $\left[\mathrm{Pd}\left(\mathrm{k}^{2}-\mathrm{dppp}\right)\right.$ $\mathrm{Cl}_{2}$ ] complex ${ }^{30,31}$. The crystal determination data and selected bond distance and bond angles for $\left[\mathrm{Pd}\left(\mathrm{k}^{2}-\mathrm{dppp}\right) \mathrm{Cl}_{2}\right]$ complex is summarized in Tables 4 and 6 .

The $\left[\mathrm{Pt}\left(\mathrm{k}^{2}-\mathrm{dppmCl}\right) \mathrm{Cl}_{2}\right]$ complex was crystallized from a $\mathrm{CH}_{2} \mathrm{Cl}_{2}$ solution of the complex at room temperature, after a five days, a yellow colored needle type crystals are formed, these crystals were subjected to single-crystal X-ray diffractometer study. The molecular structure of $\left[\mathrm{Pt}\left(\mathrm{k}^{2}-\mathrm{dppmCl}\right) \mathrm{Cl}_{2}\right]$ complex is shown in Fig. 3. The structural analysis reveals that $\left[\mathrm{Pt}\left(\mathrm{k}^{2}-\mathrm{dppmCl}\right) \mathrm{Cl}_{2}\right]$ complex has a square planer geometry around the metal center and that the two phosphorus and two chlorine atoms are coordinated to the platinum ion. The dppm behave as behave as bidentate chelatingligand. Whereas the Pt-P bond length of 2.217(2) and 2.217(2) $\AA$ for $\mathrm{P}(1)$ and $\mathrm{P}(2)$ atoms of the $\left[\mathrm{Pt}\left(\mathrm{k}^{2}-\mathrm{dppmCl}\right) \mathrm{Cl}_{2}\right]$ complex ${ }^{30,31}$. The crystal determination data and selected bond distances and bond angles for $\left[\mathrm{Pt}\left(\mathrm{k}^{2}-\right.\right.$
dppmCl) $\mathrm{Cl}_{2}$ ] complex is summarized in Tables 4 and 7.

\section{CONCLUSIONS}

In this research we have describe the synthesis and characterization of some Palladium and platinum complexes obtained from the reaction of the Thioester ligand with tertiary diphosphines metal complexes as shown in Figure 1. The manner of bonding and overall structure of complexes were determined through elemental analysis, magnetic susceptibility, molar conductance, IR spectral data and UV-Visible. All synthesized complexes are square planar and diamagnetic.

\section{ACKNOWLEDGEMENTS}

The authors are thankful to the chemistry Department. College of Education-Salahaddin University for their accomplishing our present work and Prof T.I.A. Gerberand Eric. C. Hosten at Nelson Mandela Metropolitan Universityfor their help in obtaining the X-ray crystallographic data.

\section{REFERENCES}

1. Walia, R;Hedaitullah, M;Naaz, S.F.; Iqbal, K;Lamba, H. Int. J. Res. Pharm. Chem. 2011,3, 565-574

2. Vikash, K;Deveder, P.Int. Res. J. of Pharm. 2014, 12, 861-875

3. Husain, A; Varshney, M; Rashid, M; Mishra, R;Akhter, A. J. of Pharm. Res.2011,2, 413419

4. Alias, M;Mahal, D. Int. J. of Sci. and Res.2015,7, 1469-1476

5. Finšgar, M.Corr. Sci. 2013,72, 90-98

6. Anandara, K;Tiwari, R;Bothara, K;Sunilson, J;Dineshkumar, C;Promwichit, P.Adv. in App. Sci. Res. 2010,2, 132-138

7. Mor, M;Bordi, F; Silva, C;Rivara, S;Zuliani, V;Vacondio, F;Rivara, M;Barocelli, E;Bertoni, S;Ballabeni, V.Bioorg. Medi. Chem. 2004, 4,663-674

8. Bakhareva, E; Voronkov, M; Sorokin, M;Lopyrev, V;Seredenin, S;Gaidarov, G.Pharm. Chem. J.1996, 2,89-91
9. Anandara, K;Tiwari, R;Venkateshan, N;Pooshan, G;Promwichit, P.J. Chem and Pharm res. 2010,3, 230-236

10. Thakuria, H; Das, G. Arkivoc. 2008,15, 321328

11. Lobana, T; Sultana, R. J. Chem. Sci.2012,6, 1261-1268

12. Kazemi, M;Shiri, L.J. Sulf. Chem. 2015,6,111

13. Pourshahbaz, M;Irandoust, M;Rafiee, E;Joshaghani, M. Polyhedron. 2009,3, 609613

14. Cavell, R.Curr. Sci. 2000,4, 441-452

15. Lassahn, P;Lozan, V; Weller, A;Janiak, C.Dalton Trans. 2003,23, 4437-4450

16. APEX; SADABS; SAINT.2010, Bruker AXS Inc., Madison, Wisconsin, USA.

17. Sheldrick, G.M. ActaCrystallogr. 2008, A 64, 112-122

18. Hubschle, C. B.;Sheldrick, G.M.;Dittrich, B.J App/Cryst. 2011, 44, 1281-1284 
19. Enaam, M. R.Diyala J. for Pure Sci. 2013,1, 97-110

20. Pessoa, J; Duarte, M; Gillard, R; Madeira, C;Matias, P;Tomaz, I.J. ChemSoc, Dalton Trans. 1998, 23, 4015-4020

21. Amin, O; Al-Hayaly, L; Al-Jibori, S; Al-Allaf, T.Polyhedron. 2004, 11, 2013-2020

22. Bu'itrus, N;Hussain, K.Asian J. of Chem. 2003, 4, 1617-1622

23. Al-Jibori, S; Al-Jibori, M; Hogarth, G. Inorg. Chim.Acta.2013, 398, 117-123

24. Al-Hayaly, L; Abdullah, B. H.; Al-Dulaimi, A; Al-Jibori, S. A. Oriental J. of Chem. 2008, 2, 381-388
25. Edwards.P;Jaouhari, R.Polyhedron.1989, 1, 25-28

26. Kumbhare, L; Jain, V; Varghese, B.Inorg. Chim.Acta.2006, 2, 409-416

27. Sutton, D. 1968 McGraw-Hill 192-

28. Pastorek, R;Kameníèek, J;Krystýnková, P;Šindeláø, Z.ActaUniversitatisPalackianae Olomucensis.2000,39, 63

29. Al-Jibori, S; Abdullah, A; Al-Allaf, T. Tran. Metal Chem. 2007, 3,398

30. Al-Jibori, S; Al-Jibori, Q; Schmidt, H;Merzweiler, K; Wagner, C; Hogarth, G.Inorg. Chim. Acta.2013, 402, 69-74

31. Steffen, W;Palenik, G.Inorg. Chem. 1976, 10, 2432-2439 